\title{
Editorial
}

\section{Laser interstitial thermal therapy: an effective treatment for focally recurrent high grade glioma}

Hani R. Malone, M.D., and Jeffrey N. Bruce, M.D.

Department of Neurological Surgery, Columbia University, New York, New York

Laser interstitial thermal therapy (LITT) is an emerging treatment modality in glioma therapy. The technology is most effectively applied in the treatment of deep-seated lesions that are difficult to access surgically without injury to eloquent neurological structures. The LITT treatment produces focal thermal ablation leading to tumor cytoreduction through tissue coagulation, necrosis, and cellular apoptosis. Historically, laser ablation techniques have been limited by an inability to assess ablation progress and parenchymal temperature during the course of treatment. Advances in MRI capabilities have overcome these limitations, leading to the reapplication of this technology for select intracranial pathological entities, with promising preliminary results.

In this issue of Neurosurgical Focus, Hawasli and colleagues review the current literature on LITT for the treatment of high-grade gliomas (HGGs). ${ }^{3}$ This report underscores the variability in published outcomes following LITT for HGG. Radiographically, the reported reduction in tumor size on follow-up imaging after LITT varies between $15 \%$ and $87 \%$ of total lesion volume. ${ }^{2,4}$ The average progression-free survival post-LITT ranges from 1 to 17 months, while overall survival post-LITT varies from 7 to 30 months. ${ }^{3}$ This variability may in part be attributed to mixed patient cohorts, including both newly diagnosed and recurrent tumors.

Treatment results appear more consistent when limited to patients with disease recurrence, although this subgroup analysis is not available in all trials. Four studies that evaluated LITT as salvage therapy for recurrent HGG reported an overall survival of 9-15 months posttreatment. ${ }^{1,5,7,8}$ Although these promising data are similar to survival achieved with salvage surgery for HGG, it is impossible to control for the contribution of adjuvant chemotherapies to this outcome. Nevertheless, recurrence may represent a favorable context for LITT application, because patients in this cohort are often poor surgical can- didates with focal disease. Data from subgroup analysis of patients treated for newly diagnosed HGG are scarce. The varying indications that led to LITT as de novo treatment in these instances further limit conclusions that can be drawn from these data.

Consistent with the surgical literature on extent of resection in HGG, "extent of ablation" appears to be an important factor influencing both overall and progressionfree survival. ${ }^{6}$ Additionally, increasing the thermal dose appears to be associated with improved radiographic response and perhaps clinical outcome. ${ }^{2,8}$ Intuitively, dose escalation with more aggressive laser ablation will increase the risk profile associated with LITT, particularly with the propensity for blood vessels and ventricles to act as heat sinks. Hawasli and colleagues report an overall complication rate of $49 \%$ after pooling data in their review of 174 HGG cases. Although some of these complications are minor and transient, there were 4 reports of cerebral edema, 30 of neurological deficits, and 6 reports of infection.

Data on the comparative safety and efficacy of stereotactic laser ablation are limited by inherent selection bias for patients with difficult tumor locations, lower performance status, and poor eligibility for open surgery. Interpretation of pooled outcome data is further complicated by varying reporting measures and diverse adjuvant treatment regimens. Accordingly, Hawasli and colleagues emphasize the incorporation of LITT into standard practice algorithms to generate case-controlled data that will allow for a more sound assessment of the technology. Additional studies incorporating genetic and molecular data (i.e., tumor subtype, methylguanine methyltransferase [MGMT] methylation, and isocitrate dehydrogenase-1 [IDH-1] mutation) may further define which lesions are most appropriate for LITT. Well-circumscribed focal lesions may be well treated, whereas LITT is unlikely to achieve adequate cytoreduction in diffuse, invasive tumors. A better understanding of the effects of LITT-induced blood-brain barrier breakdown on chemotherapeutic drug delivery will also inform the appropriate use of this technology moving forward. Currently, stereotactic laser ablation for HGG seems most appropriate for cases of focal recurrence and as an adjunct to stereotactic biopsy with subsequent adjuvant treatment. Although the results to date are provocative and warrant further study, there is insufficient evidence to support this modality as an alternative to maximal safe resection with open surgery.

(http://thejns.org/doi/abs/10.3171/2014.9.FOCUS14671) 


\section{Editorial}

\section{Disclosure}

The authors report no conflict of interest.

\section{References}

1. Carpentier A, Chauvet D, Reina V, Beccaria K, Leclerq D, McNichols RJ, et al: MR-guided laser-induced thermal therapy (LITT) for recurrent glioblastomas. Lasers Surg Med 44:361-368, 2012

2. Hawasli AH, Bagade S, Shimony JS, Miller-Thomas M, Leuthardt EC: Magnetic resonance imaging-guided focused laser interstitial thermal therapy for intracranial lesions: single-institution series. Neurosurgery 73:1007-1017, 2013

3. Hawasli AH, Kim AH, Dunn GP, Tran DD, Leuthardt EC: Stereotactic laser ablation of high-grade gliomas. Neurosurg Focus 37(6):E1, 2014

4. Kahn T, Bettag M, Ulrich F, Schwarzmaier HJ, Schober R, Fürst G, et al: MRI-guided laser-induced interstitial thermotherapy of cerebral neoplasms. J Comput Assist Tomogr 18: 519-532, 1994

5. Leonardi MA, Lumenta CB: Stereotactic guided laser-in- duced interstitial thermotherapy (SLITT) in gliomas with intraoperative morphologic monitoring in an open MR: clinical expierence. Minim Invasive Neurosurg 45:201-207, 2002

6. Mohammadi AM, Hawasli AH, Rodriguez A, Schroeder JL, Laxton AW, Elson P, et al: The role of laser interstitial thermal therapy in enhancing progression-free survival of difficult-toaccess high-grade gliomas: a multicenter study. Cancer Med 3:971-979, 2014

7. Schwarzmaier HJ, Eickmeyer F, Tempelhoff von W, Fiedler VU, Niehoff H, Ulrich SD, et al: MR-guided laser-induced interstitial thermotherapy of recurrent glioblastoma multiforme: preliminary results in 16 patients. Eur J Radiol 59:208-215, 2006

8. Sloan AE, Ahluwalia MS, Valerio-Pascua J, Manjila S, Torchia MG, Jones SE, et al: Results of the NeuroBlate System first-in-humans Phase I clinical trial for recurrent glioblastoma. Clinical article. J Neurosurg 118:1202-1219, 2013

Please include this information when citing this paper: DOI: 10.3171/2014.9.FOCUS14671. 